\title{
PERIODIC AND FIXED POINT THEOREMS IN A QUASI-METRIC SPACE
}

\section{LJUBOMIR ĆIRIĆ}

(Received 6 November 1990)

Communicated by J. H. Rubinstein

\begin{abstract}
General periodic and fixed point theorems are proved for a class of self maps of a quasi-metric space which satisfy the contractive definition (A) below. Two examples are presented to show that the class of mappings which satisfy $(A)$ is indeed wider than a class of selfmaps which satisfy Caristi's contractive definition (C) below. Also a common fixed point theorem for a pair of maps which satisfy a contractive condition (D) below is established.
\end{abstract}

1991 Mathematics subject classification (Amer. Math. Soc.): 54 H 25, 47 H 10.

\section{Introduction}

Let $X$ be a non-void set and $T: X \rightarrow X$ a selfmap. A point $x \in X$ is called a periodic point for $T$ iff there exists a positive integer $k$ such that $T^{k} x=x$. If $k=1$, then $x$ is called a fixed point for $T$.

Caristi [4] proved the following very general contraction fixed point theorem.

TheOREM 1 (Caristi [4]). Suppose $T: X \rightarrow X$ and $\Phi: X \rightarrow[0, \infty)$, where $X$ is a complete metric space and $\Phi$ is lower semicontinuous. If for each $X$ in $X$

$$
d(x, T x) \leq \Phi(x)-\Phi(T x),
$$

then $T$ has a fixed point.

(C) 1993 Australian Mathematical Society $0263-6115 / 93 \$ A 2.00+0.00$ 
Caristi's proof, based on the work of Brondsted [3], is not elementary, as well as the other new proofs of Theorem $1([10,13])$. Bhakta and Basu [1] observed that the proof of Theorem 1 becomes much simpler by adding the hypotheses of orbital continuity of a selfmap $T$ (compare [5]). Moreover, they pointed out that the hypotheses of the lower semi-continuity of a function $\Phi$ in that case may be dropped.

Recently Bollenbacher and Hicks [2] obtained a version of Caristi's Theorem 1 by using the concept of $T$-orbitally lower semi-continuity of a real function $G: X \rightarrow[0, \infty)$ (defined by $G(x)=d(x, T x)$ ), which was introduced in [9] (compare [12]). Hicks [8] extended this version for a metric space to one for a quasi-metric space, which need not satisfy $d(y, x)=d(x, y)$.

The purpose of this note is to relax Caristi's contractive definition (C), slightly relax the concept of $T$-orbital lower semi-continuity introduced in [9] and to obtain a periodic and a fixed point theorem which extend and generalize main results of [2], [7] and [8]. We shall also prove a common fixed point theorem having the fixed point theorems of $[2,7,8]$ as corollaries.

\section{Main results}

Let $(X, d)$ be a quasi-metric space and $T: X \rightarrow X$ a mapping of $X$. A set $0(x, \infty)=\left\{x, T x, T^{2} x, \ldots,\right\}$ is called the orbit of $x$.

Definition 2.1. A real-valued function $G: X \rightarrow[0, \infty)$ is said to be $T$-orbitally weak lower semi-continuous (w.l.s.c.) relative to $x$ iff $\left\{x_{n}\right\}$ is a sequence in $0(x, \infty)$ and

$$
\lim _{n \rightarrow \infty} x_{n}=p \text { implies } G(p) \leq \lim _{n \rightarrow \infty} \sup G\left(x_{n}\right) .
$$

Clearly, every function $G$ that is $T$-orbitally lower semi-continuous (l.s.c.) relative to $x \in X$ (that is, $\left\{x_{n}\right\} \subseteq 0(x, \infty)$ and $\lim x_{n}=p$ imply $G(p) \leq$ $\lim \inf G\left(x_{n}\right)$ (see $\left.[9,2]\right)$ ) is also $T$-orbitally w.l.s.c. relative to $x$, but the implication is not reversible.

Note that the condition (1) was used in [6], but there it was supposed that (1) was true for every sequence $\left\{x_{n}\right\}$ in $X$.

Theorem 2. Suppose $T: X \rightarrow X, n: X \rightarrow N$ and $\Phi: X \rightarrow[0, \infty)$, where $X$ is a complete quasi-metric space. If for some $x_{0} \in X$ there exists a subsequence $S=\left\{x_{n}\right\}_{n=0}^{\infty}$ in $0\left(x_{0}, \infty\right)$ such that $T^{n\left(x_{n}\right)} x_{n} \in S$ and

$$
d\left(y, T^{n(y)} y\right) \leq \Phi(y)-\Phi\left(T^{n(y)} y\right)
$$

holds for each $y \in S$, then we have

(a) $\lim x_{n}=p$ exists, 
(b) $T^{n(p)} p=p$ if and only if $G(x)=d\left(x, T^{n(x)} x\right)$ is $T$-orbitally w.l.s.c. relative to $x_{0}$,

(c) $d\left(x_{0}, x_{n}\right) \leq \Phi\left(x_{0}\right)$,

(d) If $y \rightarrow d(z, y)$ is $T$-orbitally w.l.s.c. relative to $x_{0}$ for $z \in S$ then $d\left(x_{n}, p\right) \leq \Phi\left(x_{n}\right)$ and $d\left(x_{0}, p\right) \leq \Phi\left(x_{0}\right)$.

Proof. Without loss of generality we may suppose that $\left\{x_{n}\right\}$ has the property that $x_{n+1}=T^{n\left(x_{n}\right)} x_{n} ; n=0,1,2, \ldots$. Then we have, for $n=$ $0,1,2, \ldots$,

$$
d\left(x_{n}, x_{n+1}\right)=d\left(x_{n}, T^{n\left(x_{n}\right)} x_{n}\right) \leq \Phi\left(x_{n}\right)-\Phi\left(x_{n+1}\right) .
$$

For $m \geq 0$,

$$
\begin{aligned}
\sigma_{m}= & \sum_{n=0}^{m} d\left(x_{n}, x_{n+1}\right) \leq \sum_{n=0}^{m}\left[\Phi\left(x_{n}\right)-\Phi\left(x_{n+1}\right)\right]=\left[\Phi\left(x_{0}\right)-\Phi\left(x_{1}\right)\right] \\
& +\left[\Phi\left(x_{1}\right)-\Phi\left(x_{2}\right)\right]+\cdots+\left[\Phi\left(x_{m}\right)-\Phi\left(x_{m+1}\right)\right] \\
= & \Phi\left(x_{0}\right)-\Phi\left(x_{m+1}\right) \leq \Phi\left(x_{0}\right) .
\end{aligned}
$$

The sequence $\left\{\sigma_{m}\right\}_{m=0}^{\infty}$ of partial sums of the infinite series $\sum d\left(x_{n}, x_{n+1}\right)$ is a nondecreasing sequence bounded above by $\Phi\left(x_{0}\right)$ and therefore converges. This implies that $\left\{x_{n}\right\}_{n=0}^{\infty}$ is a Cauchy sequence in $X$. Since $(X, d)$ is complete, we have (a).

Assume that $G(p) \leq \lim _{n \rightarrow \infty} \sup G\left(x_{n}\right)$. Then by definition of $G(x)$ and $x_{n+1}$ we have $G\left(x_{n}\right)=d\left(x_{n}, x_{n+1}\right)$. So $G\left(x_{n}\right)$ is a general term of a convergent series $\sum d\left(x_{n}, x_{n+1}\right)$, and hence $G\left(x_{n}\right) \rightarrow 0$, as $n \rightarrow \infty$. Therefore, $G(p)=d\left(p, T^{n(p)} p\right)=0$. Hence $p$ is a periodic point of $T$. This shows (b). Clearly (c) holds.

To prove (d), let $n \geq 0$. Then

$$
\begin{aligned}
d\left(x_{n}, x_{n+k}\right) & \leq d\left(x_{n}, x_{n+1}\right)+d\left(x_{n+1}, x_{n+2}\right)+\cdots+d\left(x_{n+k-1}, x_{n+k}\right) \\
& =\sum_{m=n}^{n+k-1} d\left(x_{m}, x_{m+1}\right) \leq \sum_{m=n}^{n+k-1}\left[\Phi\left(x_{m}\right)-\Phi\left(x_{m+1}\right)\right] \\
& =\Phi\left(x_{n}\right)-\Phi\left(x_{n+k}\right) \leq \Phi\left(x_{n}\right) .
\end{aligned}
$$

Assume that $y \rightarrow d\left(x_{n}, y\right)$ is $T$-orbitally w.l.s.c. relative to $x_{0}$ for each $n=0,1,2, \ldots$. Then

$$
d\left(x_{n}, p\right) \leq \lim _{k \rightarrow \infty} \sup d\left(x_{n}, x_{n+k}\right) \leq \lim _{k \rightarrow \infty} \sup \Phi\left(x_{n}\right)=\Phi\left(x_{n}\right) .
$$

This shows (d).

REMARK 1. Example 1 shows that in (b) need not be $n(p)=1$, that is, $T$ need not have a fixed point. 
Theorem 3. Suppose $T: X \rightarrow X, n: X \rightarrow N$ and $\Phi: X \rightarrow[0, \infty)$, where $X$ is a complete quasi-metric space. If $T$ satisfies all hypotheses of Theorem 2 and in addition for all $y \in C l\left[0\left(x_{0}, \infty\right)\right]$

$$
y \neq T y \text { implies } \Phi\left(T^{m} y\right)<\Phi(y)
$$

for some positive integer $m=m(y)$, then $T$ has a fixed point.

Proof. From Theorem 2, there is $p$ in $X$ such that $T^{k} p=p$. Then $0(p, \infty)$ is a finite set of points in $X$. Let $y \in 0(p, \infty)$ be such that

$$
\Phi(y)=\min \{\Phi(z): z \in 0(p, \infty)\} .
$$

Assume that $y \neq T y$. Then from (1) there is $m \in N$ such that $\Phi\left(T^{m} y\right)<$ $\Phi(y)$. But, since $T^{n} y \in 0(p, \infty)$ for all $n \in N$, it follows from (2) that $\Phi(y) \leq \Phi\left(T^{m} y\right)$, a contradiction. Therefore, $y=T y$, which completes the proof.

Corollary 1 (Hicks [8, Theorem 2]). Let $X$ and $Y$ be quasi-metric spaces with $X$ complete. Suppose $T: X \rightarrow X, f: X \rightarrow Y$ and $\Phi: f X \rightarrow$ $[0, \infty)$. If there exists $x_{0} \in X$ and $c>0$ such that

$$
\max \{d(y, T y), c \cdot d(f y, f T y)\} \leq \Phi(f y)-\Phi(f T y)
$$

for all $y \in 0\left(x_{0}, \infty\right)$, then

(a') $\lim T^{n} x_{0}=p$ exists,

$\left(\mathrm{b}^{\prime}\right) T p=p$ if and only if $G(x)=d(x, T x)$ is $T$-orbitally l.s.c. relative to $x_{0}$,

(c') $d\left(x_{0}, T^{n} x_{0}\right) \leq \Phi\left(f x_{0}\right)$,

(d) If $y \rightarrow d(z, y)$ is continuous for $z \in 0\left(x_{0}, \infty\right)$, then $d\left(T^{n} x_{0}, p\right) \leq$ $\Phi\left(f T^{n} x_{0}\right)$ and $d\left(x_{0}, p\right) \leq \Phi\left(f x_{0}\right)$.

Proof. It is clear that (B) implies that $d(y, T y) \leq \Phi(f y)-\Phi(f T y)$. Put $\Phi_{1}=\Phi f$. Then $\Phi_{1}: X \rightarrow[0, \infty)$ and

$$
d(y, T y) \leq \Phi_{1}(y)-\Phi_{1}(T y) .
$$

Therefore, if $T$ satisfies (B), then $T$ satisfies (A) and (1) with $\Phi=\Phi_{1}$, $n(y)=1$ and $m(y)=1$ for all $y \in S=0\left(x_{0}, \infty\right)$.

Remark 2. Example 2 shows that Theorem 3 is a proper generalization of Hick's theorem [8], which is an extension of corresponding theorems for metric spaces given in $[2,7]$.

REMARK 3. The proof of Corollary 1 shows that the condition $c d(f x, f T x)$ $\leq \Phi(f x)-\Phi(f T x)$ in Theorems 2 and 3 in [7] and in Theorem 2 in [8] (that 
is, in our Corollary 1), which is included in (B), can be dropped. Theorem 2 and its Corollary 1 in [8] are equivalent.

Now we shall prove a common fixed point theorem for two maps.

Theorem 4. Suppose $S, T: X \rightarrow X$ and $\Phi: X \rightarrow[0, \infty)$, where $X$ is a complete quasi-metric space. If there is $x_{0} \in X$ such that

$$
d(y, T y)+d(T y, S T y) \leq \Phi(y)-\Phi(S T y)
$$

for all $y \in 0_{S T}\left(x_{0}, \infty\right)=\left\{x_{0}, T x_{0}, S T x_{0}, T(S T) x_{0}, \ldots,(S T)^{n} x_{0}\right.$, $\left.T(S T)^{n} x_{0}, \ldots\right\}$, then we have

$\left(\mathrm{a}^{\prime \prime}\right) \lim _{n \rightarrow \infty}(S T)^{n} x_{0}=\lim _{n \rightarrow \infty} T(S T)^{n} x_{0}=p$ exists.

$\left(\mathrm{b}^{\prime \prime}\right) T p=p$, if $G_{1}(x)=d(x, T x)$ is $(S, T)$-orbitally w.l.s.c. relative to $x_{0}$ (that is, (1) is true if $\left\{x_{n}\right\} \subseteq 0_{S T}\left(x_{0}, \infty\right)$ ).

( $\left.{ }^{\prime \prime}\right) T p=p=S p$, if $G_{1}(x)=d(x, T x)$ and $G_{2}(x)=d(x, S x)$ are $(S, T)$-orbitally w.l.s.c. relative to $x_{0}$.

Proof. Put $z_{2 k}=(S T)^{k} x_{0}, z_{2 k+1}=T z_{2 k}(k=0,1,2, \ldots$,$) and con-$ sider the sequence $\left\{z_{n}\right\}_{n=0}^{\infty}$. Just as in the proof of (a) of Theorem 2, by (D) we obtain $\sum_{n=0}^{\infty} d\left(z_{n}, z_{n+1}\right) \leq \Phi\left(x_{0}\right)$. Hence $\lim _{n \rightarrow \infty} z_{n}=p$ exists. Hence $\lim _{k \rightarrow \infty} z_{2 k}=\lim _{k \rightarrow \infty}(S T)^{k} x_{0}=p$ and $\lim _{k \rightarrow \infty} z_{2 k+1}=\lim _{k \rightarrow \infty} T(S T)^{k} x_{0}$ $=p$. This shows (a"). Since $z_{2 k} \rightarrow p$ and $G_{1}\left(z_{2 k}\right)=d\left(z_{2 k}, z_{2 k+1}\right) \rightarrow 0$ as $k \rightarrow+\infty$, we have $G_{1}(p)=0$. Hence $T p=p$. This shows $\left(\mathrm{b}^{\prime \prime}\right)$. Statement $\left(c^{\prime \prime}\right)$ clearly holds.

\section{Examples}

1. Let $X=[-2,-1] \cup[1,2]$ with the usual metric. Define $T: X \rightarrow X$ by $T x=-x$ and $\Phi: X \rightarrow[0, \infty)$ by $\Phi(x)=|x|$, for example. Then $T$ satisfies (A) for all $y \in X$ with $n(y)=2$ and $G(x)=d\left(x, T^{2} x\right)$ is continuous on $X$.

2. Let $X=\{0\} \cup\{ \pm 1 / n: n=1,2, \ldots\}$ with the usual metric. Define $T: X \rightarrow X$ by $T(1 / n)=-1 /(n+1), T(-1 / n)=1 /(n+1)$ and $T(0)=0$. Define $\Phi: X \rightarrow[0, \infty)$ by $\Phi(x)=d(x, T x)$. Then for $x= \pm 1 / n$ we have

$$
d(x, T x)=1 / n+1 /(n+1) ; \quad d\left(x, T^{2} x\right)=1 / n-1 /(n+2) .
$$

Hence

$$
\begin{aligned}
d\left(x, T^{2} x\right) & =1 / n-1 /(n+2)<1 / n+1 /(n+1)-[1 /(n+2)+1 /(n+3)] \\
& =\Phi(x)-\Phi\left(T^{2} x\right)
\end{aligned}
$$


Therefore, $T$ satisfies (A) on $X$ with $n(x)=2$ for all $x \in X$. Since $X$ is a complete metric space, there exists $p(p=0)$ such that (a) holds. Since $G(x)=d\left(x, T^{2} x\right)=2 x^{2}(1+2|x|)^{-1}$ is continuous and $T$ satisfies (1) with $m(y)=2$ for all $y \in X$, Theorem 3 can be applied.

We point out that Caristi's contractive condition (C), and hence (B), implies that $\sum_{n=0}^{\infty} d\left(T^{n} x, T T^{n} x\right)$ must be a convergent series. Since in our example, for any fixed $x= \pm 1 / m_{0}$, we have

$$
d\left(T^{n} x, T^{n+1} x\right)=1 /\left(n+m_{0}\right)+1 /\left(n+1+m_{0}\right)>2 /\left(n+m_{0}+1\right),
$$

we conclude that the series diverges and so there is no functions $f: X \rightarrow Y$ and $\Phi: f X \rightarrow[0, \infty)$ such that $(B)$ holds for any $x= \pm 1 / n \in X$.

\section{References}

[1] P. S. Bhakta and T. Basu, 'Some fixed point theorems on metric spaces', J. Indian Math. Soc. 45 (1981), 399-404.

[2] A. Bollenbacher and T. L. Hicks, 'A fixed-point theorem revisited', Proc. Amer. Mat. Soc. 102 (1988), 898-900.

[3] A. Brondsted, 'On a lemma of Bishop and Phelps', Pacific J. Math. 55 (1974), 335-341.

[4] J. Caristi, ' Fixed point theorems for mappings satisfying inwardness conditions', Trans. Amer. Math. Soc. 215 (1976), 241-251.

[5] Lj. Cirić, 'On contraction type mappings', Math. Balkanica 1 (1971), 52-57.

[6] Lj. Ciric, 'On mappings with a contractive iterate', Publ. Inst. Math. (Beograd), 26 (40) (1979), 79-82.

[7] A. Harder and T. L. Hicks, 'Fixed point theory and iteration procedures', Indian J. Math. 19 (1988), 17-26.

[8] T. L. Hicks, 'Fixed point theorems for quasi-metric spaces', Math. Japon. 33 (1988), 231-236.

[9] T. Hicks and B. Rhoades, 'A Banach type fixed point theorem', Math. Japon. 24 (1979), 327-330.

[10] J. Siegel, 'A new proof of Caristi's fixed point theorem', Proc. Amer. Math. Soc. 66 (1977), 54-56.

[11] R. A. Stoltenberg, 'On quasi-metric spaces', Duke Math. J. 36 (1969), 65-71.

[12] C. S. Wong, 'Fixed point theorems for non-expansive mappings', J. Math. Anal. Appl. 37 (1972), 142-150.

[13] C. S. Wong, 'On a fixed point theorem of contractive type', Proc. Amer. Math. Soc. 57 (1976), 283-284.

\section{Matematički Institut}

Kneza Mihaila 35

11000 Beograd

Yugoslavia 